\title{
Determination of paramagnetic and ferromagnetic phases of an Ising model on a third-order Cayley tree
}

\author{
H. Akın $]^{k}$ \\ Ceyhun Atuf Kansu Caddesi 1164. Sokak, 9/4, TR06105, Çankaya, Ankara, Turkey
}

Received March 28, 2020, in final form September 23, 2020

In this present paper, the recurrence equations of an Ising model with three coupling constants on a thirdorder Cayley tree are obtained. Paramagnetic and ferromagnetic phases associated with the Ising model are characterized. Types of phases and partition functions corresponding to the model are rigorously studied. Exact solutions of the mentioned model are compared with the numerical results given in Ganikhodjaev et al. [ J. Concr. Appl. Math., 2011, 9, No. 1, 26-34].

Key words: Cayley tree, Ising model, paramagnetic phase, ferromagnetic phase

\section{Introduction}

In magnetic and ferroelectric systems, phase diagrams of a model with various transition lines and modulated phases are obtained with the presence of different competing interactions [1]. In order to picture all phase diagrams of a given model, many researchers used the recurrence relations associated with the model and observed their dynamical properties after a large number of iterations [2, 6]. Recently, in references [2, 5, 7], some features of complex phase diagrams corresponding to the ANNNI (Axial Next-Nearest-Neighbor Ising) model consisting of an Ising spin Hamiltonian on a Cayley tree were studied.

The existence and quantification of the modulated phase diagrams after iterations of relevant recurrence equations as a way of probing the ground phases of Ising model have gained much attention [1, 2, 4, 5, 8,-16]. In general, many authors have plotted the phase diagrams associated with the model by means of the periodic fixed points of an operator consisting of recurrent relations [2, 4, 5, 8. 12, 17]. In the above-mentioned works, most results are numerically obtained. In [18], Chakraborty investigated the effects of inclusion of three-spin and four-spin couplings to the Ising model. Furthermore, Chakraborty employed the molecular-field approximation to study the effects of both three-spin and four-spin couplings on the field-free and field-induced phase transitions possible for the model. In this paper, we obtain our results in an analytical way by comparing the numerical results.

Horiguchi [19] proved that there exists a phase transition in the Ising model on the square lattice with two-spin interactions in the vertical direction and with slantwise, alternate three-spin interactions in the horizontal direction. Moreover, Horiguchi proved that the system described by Hamiltonian is in the paramagnetic state at high temperatures and obtained an upper bound to the critical temperature. Azhari et al. [20] investigated the magnetic properties of the mixed spin-1/2 and spin-1 Ising ferromagnetic system with four-spin interaction $J_{4}$ and next-nearest neighbor (NNN) coupling $J_{0}$. They are interested in the phase diagram and in the location and the multitude of the compensation point.

In [4], we numerically studied the Lyapunov exponent and modulated phases for the Ising model with different coupling constants on an arbitrary-order Cayley tree. We also plotted the variation of the wavevector $q$ with temperature in the modulated phases. In [21] we described the existence of a phase transition problem by means of Gibbs measures of the Potts model on an order three Cayley tree. In [22],

\footnotetext{
*email: akinhasan25@ gmail.com
} 


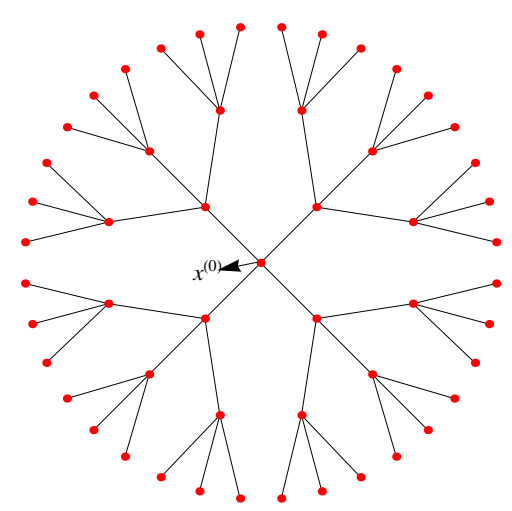

Figure 1. (Colour online) Three-order Cayley tree with three levels.

we constructed a class of new Gibbs measures by extending the known Gibbs measures for the Ising model on a Cayley tree of the order $k_{0}$ to a Cayley tree of higher order $k>k_{0}$. Nazarov and Rozikov established the invariant subsets of an operator given in [23] and identified the periodic Gibbs measures with period two by means of the operator. Here, in order to obtain the periodic fixed points associated with the recurrence equations, we use similar methods in [24, 25].

In [26], the author studied the Gibbs measures associated with Vannimenus-Ising model for the compatible conditions. He proved the existence of the translation-invariant Gibbs measures with respect to the compatible conditions.

In [26-29], we considered an external magnetic field to investigate the Gibbs measures (see [30, 31]). In [32], we analytically studied the recurrence equations of an Ising model with two coupling constants on a second-order Cayley tree without considering the numerical investigation. Therefore, our present results differ from the ones obtained in the mentioned papers.

The main purpose of the paper is to analytically derive the recurrence equations of an Ising model with three coupling constants on a third-order Cayley tree and to obtain the paramagnetic, ferromagnetic and 2-period phases of the model by means of the related recurrence equations by using the method given in [32]. We exactly describe the paramagnetic phase of the Ising model. We investigate some phase diagrams of an Ising model with 2-spin couplings between the nearest neighbors and the next-nearest neighbors, plus a 3-spin interaction, on a Cayley tree with coordination number $z=4$. We obtain some rigorous results: critical temperatures and curves, number of phases, the partition functions. This model was numerically examined by Ganikhodjaev et al. [8] on semi-infinite second-order Cayley tree.

\section{Preliminary}

\subsection{Cayley tree}

Any $k(k>1)$-order Cayley tree $\Gamma^{k}$ is a weave pattern in which $(k+1)$ edges from each vertex point extend infinitely as shown in figure $1(k=3)$. For the Cayley tree shown as $\Gamma^{k}=(V, \Lambda), V$ denotes the vertices of the Cayley tree and $\Lambda$ denotes the set of edges. If there is an edge $\ell$ joining two vertices $x$ and $y$, it is called "nearest neighbor" and $\ell=<x, y>$. The distance of $x$ and $y$, over $V$ is defined as $d(x, y)$, the shortest path between $x$ and $y$. The set of edge points in $V_{n}$ is represented as $L_{n}$ (see [26] for details). Note that we consider a semi-infinite third-order Cayley tree which has got uniformly bounded degrees. The set of all vertices with the distance $n$ from the root $x^{(0)}$ is called the $n$th level of $\Gamma^{k}$ and we denote the sphere of radius $n$ on $V$ by

$$
W_{n}=\left\{x \in V: d\left(x, x^{(0)}\right)=n\right\}
$$


and the ball of radius $n$ by

$$
V_{n}=\left\{x \in V: d\left(x, x^{(0)}\right) \leq n\right\} .
$$

The set of direct successors of any vertex $x \in W_{n}$ is denoted by

$$
S(x)=\left\{y \in W_{n+1}: d(x, y)=1\right\} .
$$

Hereafter, we use the following definitions for neighborhoods.

Definition 2.1. 1. Two vertices $x$ and $y, x, y \in V$ are called nearest-neighbors $(N N)$ if there exists an edge $\ell \in \Lambda$ connecting them, which is denoted by $\ell=\langle x, y\rangle$.

2. The next-nearest-neighbor vertices $x \in W_{n}$ and $z \in W_{n+2}\left(z \in S^{2}(x)\right)$ are called prolonged next-nearest-neighbors (PNNN) if $|x| \neq|z|$ and is denoted by $>x, z<$

3. The triple of vertices $x, y, z$ is called ternary prolonged next-nearest-neighbors if $x \in W_{n}, y \in S(x)$ and $z \in S(y)\left(x \in W_{n}, y \in W_{n+1}\right.$ and $\left.z \in W_{n+2}\right)$ for some nonnegative integer $n$ and is denoted by $>x, y, z<$.

In this paper, we consider a Hamiltonian with competing nearest-neighbor interactions, prolonged next-nearest-neighbors (PNNN) and ternary prolonged next nearest-neighbor interactions. Therefore, we can state the Hamiltonian by

$$
\begin{gathered}
H(\sigma)=-J \sum_{<x, y>} \sigma(x) \sigma(y)-J_{p} \sum_{>x, z<} \sigma(x) \sigma(z) \\
-J_{t} \sum_{>x, y, z<} \sigma(x) \sigma(y) \sigma(z),
\end{gathered}
$$

where $J, J_{p}, J_{t} \in \mathbb{R}$ are coupling constants and $<x, y>$ stands for NN vertices, $>x, z<$ stands for prolonged NNN and $>x, y, z<$ stands for prolonged ternary NNN.

\section{Definitions and equations}

In this section, we construct the recurrent equations associated with the Hamiltonian (2.2). It is well-known that there are different approaches for determining the Gibbs measures (phases) of lattice models such as Ising and Potts with competing interactions in the literature [29, 33-36]. Recently, some researchers [28, 29, 37, 38] determined the Gibbs measures associated with the Ising models by means of the fixed points of operators obtained by the partition functions. In our work, we use the method based on the recursive equations to derive the phases.

After specifying a Hamiltonian $H$, the equilibrium state of a physical system with Hamiltonian $H$ is described by the probability measure

$$
\mu\left(\sigma_{n}\right)=\frac{\exp \left[-\beta H\left(\sigma_{n}\right)\right]}{\sum_{\eta_{n} \in\{-1,+1\}^{V_{n}}} \exp \left[-\beta H\left(\eta_{n}\right)\right]},
$$

where $\beta$ is a positive number which is proportional to the inverse of the absolute temperature. The above $\mu$ is called the Gibbs distribution relative to $H$. The standard approach consists in writing down recurrence equations relating the partition function

$$
Z_{n}=\sum_{\eta_{n} \in\{-1,+1\}^{V_{n}}} \exp \left[-\beta H\left(\eta_{n}\right)\right],
$$

of an $n$-generation tree to the partition function $Z_{n-1}$ of its subsystems containing $(n-1)$ generations [see figure 2]. 


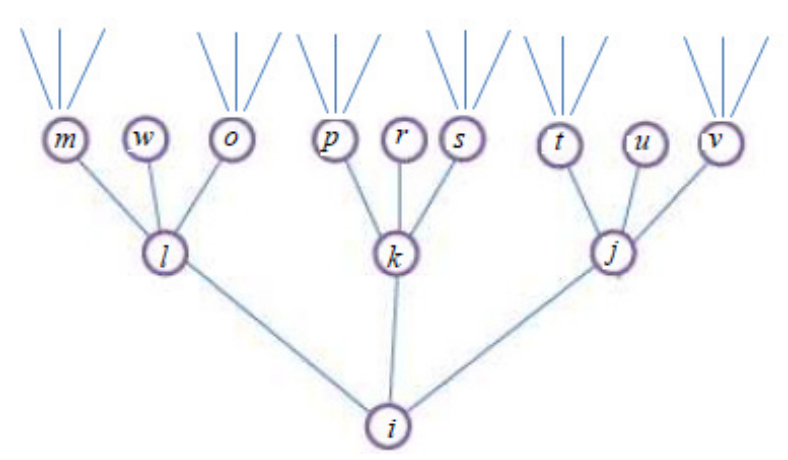

Figure 2. (Colour online) Configurations on semi-finite Cayley tree of the order three with levels 2. Schematic diagram illustrating the summation used in equation 3.1.

Many authors introduced the notions of ground states (Gibbs measure) of the Ising model with competing interactions on the Cayley tree [26, 27, 39, 40]. Here, we consider a shorter notation to write down the recurrence system

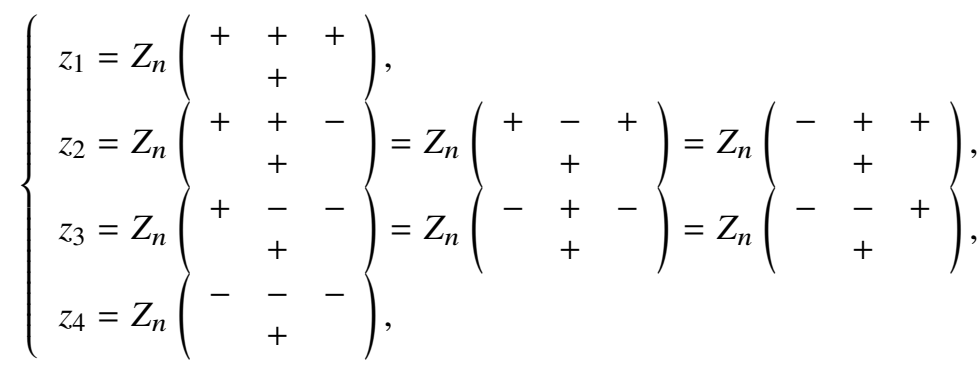

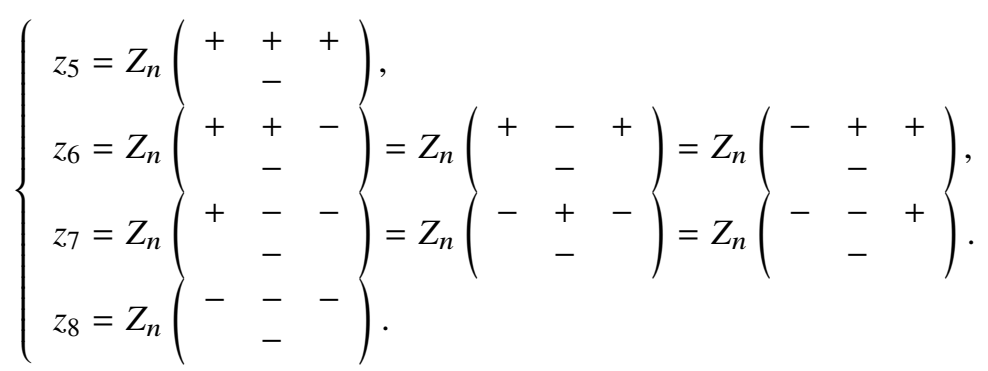

For the sake of simplicity, we denote $Z_{(n+1)}\left(\begin{array}{ccc}l & k & j \\ & i & \end{array}\right)=Z_{(n+1)}(i ; l, k, j)$. We have

$$
\begin{aligned}
& Z_{n+1}(i ; l, k, j)=\sum_{m, w, o, p, r, s, t, u, v \in\{-1,+1\}}[\exp (A(m, w, o, p, r, s, t, u, v)) \\
& \left.\times Z_{n}(l ; m, w, o) Z_{n}(k ; p, r, s) Z_{n}(j ; t, u, v)\right],
\end{aligned}
$$

where

$$
\begin{aligned}
A(m, w, o, p, r, s, t, u, v)= & J i(j+k+l)+J_{p} i(m+w+o+p+r+s+t+u+v) \\
& +J_{t}\{i[l(m+w+o)+k(p+r+s)+j(t+u+v)]\},
\end{aligned}
$$

$i, j, k, l \in\{-1,+1\}$ and $n=1,2, \ldots$ (see figure 2).

In physics, it is well known that a partition function describes the statistical properties of a system in thermodynamic equilibrium [38, 41]. Therefore, we should derive the partition functions associated with the Hamiltonian (2.2). From the equation (3.1) by using the iterative method, we can derive the partial 
partition functions as follows:

$$
\begin{gathered}
\left\{\begin{aligned}
z_{1}^{(n+1)} & =a^{3}(b c)^{-9}\left((b c)^{6} z_{1}^{(n)}+3(b c)^{4} z_{2}^{(n)}+3(b c)^{2} z_{3}^{(n)}+z_{4}^{(n)}\right)^{3} \\
z_{2}^{(n+1)} & =a(b c)^{-9}\left((b c)^{6} z_{1}^{(n)}+3(b c)^{4} z_{2}^{(n)}+3(b c)^{2} z_{3}^{(n)}+z_{4}^{(n)}\right)^{2} \\
& \times\left(b^{6} z_{5}^{(n)}+3 c^{2} b^{4} z_{6}^{(n)}+3 b^{2} c^{4} z_{7}^{(n)}+c^{6} z_{8}^{(n)}\right) \\
z_{3}^{(n+1)} & =a^{-1}(b c)^{-9}\left((b c)^{6} z_{1}^{(n)}+3(b c)^{4} z_{2}^{(n)}+3(b c)^{2} z_{3}^{(n)}+z_{4}^{(n)}\right) \\
& \times\left(b^{6} z_{5}^{(n)}+3 c^{2} b^{4} z_{6}^{(n)}+3 b^{2} c^{4} z_{7}^{(n)}+c^{6} z_{8}^{(n)}\right)^{2} \\
z_{4}^{(n+1)} & =a^{-3}(b c)^{-9}\left(b^{6} z_{5}^{(n)}+3 c^{2} b^{4} z_{6}^{(n)}+3 b^{2} c^{4} z_{7}^{(n)}+c^{6} z_{8}^{(n)}\right)^{3} \\
z_{5}^{(n+1)} & =a^{-3}(b c)^{-9}\left(z_{1}^{(n)}+3(b c)^{2} z_{2}^{(n)}+3(b c)^{4} z_{3}^{(n)}+(b c)^{6} z_{4}^{(n)}\right)^{3} \\
z_{6}^{(n+1)} & =a^{-1}(b c)^{-9}\left(z_{1}^{(n)}+3(b c)^{2} z_{2}^{(n)}+3(b c)^{4} z_{3}^{(n)}+(b c)^{6} z_{4}^{(n)}\right)^{2} \\
& \times\left(c^{6} z_{5}^{(n)}+3 c^{4} b^{2} z_{6}^{(n)}+3 c^{2} b^{4} z_{7}^{(n)}+b^{6} z_{8}^{(n)}\right) \\
z_{7}^{(n+1)} & =a(b c)^{-9}\left(z_{1}^{(n)}+3(b c)^{2} z_{2}^{(n)}+3(b c)^{4} z_{3}^{(n)}+(b c)^{6} z_{4}^{(n)}\right) \\
& \times\left(c^{6} z_{5}^{(n)}+3 c^{4} b^{2} z_{6}^{(n)}+3 c^{2} b^{4} z_{7}^{(n)}+b^{6} z_{8}^{(n)}\right)^{2} \\
z_{8}^{(n+1)} & =a^{3}(b c)^{-9}\left(c^{6} z_{5}^{(n)}+3 c^{4} b^{2} z_{6}^{(n)}+3 c^{2} b^{4} z_{7}^{(n)}+b^{6} z_{8}^{(n)}\right)^{3},
\end{aligned}\right.
\end{gathered}
$$

where $a=\mathrm{e}^{\beta J}, b=\mathrm{e}^{\beta J_{p}}, c=\mathrm{e}^{\beta J_{t}}$. Noting that

$$
\begin{aligned}
& \left(z_{2}^{(n+1)}\right)^{3}=\left(z_{1}^{(n+1)}\right)^{2} z_{4}^{(n+1)},\left(z_{3}^{(n+1)}\right)^{3}=z_{1}^{(n+1)}\left(z_{4}^{(n+1)}\right)^{2}, \\
& \left(z_{6}^{(n+1)}\right)^{3}=\left(z_{5}^{(n+1)}\right)^{2} z_{8}^{(n+1)},\left(z_{7}^{(n+1)}\right)^{3}=z_{5}^{(n+1)}\left(z_{8}^{(n+1)}\right)^{2},
\end{aligned}
$$

we obtain independent variables, introducing the new variables $u_{i}^{(n+1)}=\left(z_{i}^{(n+1)}\right)^{\frac{1}{3}}$, and we get the recurrence system of the following simpler form:

$$
\left\{\begin{array}{l}
u_{1}^{(n+1)}=\frac{a}{(b c)^{3}}\left((b c)^{2} u_{1}^{(n)}+u_{4}^{(n)}\right)^{3} \\
u_{4}^{(n+1)}=\frac{1}{a(b c)^{3}}\left(b^{2} u_{5}^{(n)}+c^{2} u_{8}^{(n)}\right)^{3} \\
u_{5}^{(n+1)}=\frac{1}{a(b c)^{3}}\left(u_{1}^{(n)}+(b c)^{2} u_{4}^{(n)}\right)^{3} \\
u_{8}^{(n+1)}=\frac{a}{(b c)^{3}}\left(c^{2} u_{5}^{(n)}+b^{2} u_{8}^{(n)}\right)^{3} .
\end{array}\right.
$$

Let us define the operator as

$$
F: u^{(n)}=\left(u_{1}^{(n)}, u_{4}^{(n)}, u_{5}^{(n)}, u_{8}^{(n)}\right) \in \mathbb{R}_{+}^{4} \rightarrow F\left(u^{(n)}\right)=\left(u_{1}^{(n+1)}, u_{4}^{(n+1)}, u_{5}^{(n+1)}, u_{8}^{(n+1)}\right) \in \mathbb{R}_{+}^{4} .
$$

Then, we can write the recurrence equations (3.2) as $u^{(n+1)}=F\left(u^{(n)}\right), n>0$ which in the theory of dynamical systems is called a trajectory of the initial point $u^{(0)}$ under the action of the operator $F$. In this way we can specify the asymptotic behavior of the partition functions $Z_{n}$ for $n \rightarrow \infty$ by means of the trajectory of $u^{(0)}$ under the action of the operator $F$. We examine the dynamical system for a given initial point $u^{(0)} \in \mathbb{R}_{+}^{4}$ in detail.

\section{Dynamics behavior of the operator $F$}

In this section we identify the fixed points of the operator $F$ given in 3.2 by using the method given in [32] and [33, chapter 3]. Here, we assume that $c=1$, or equivalently $J_{t}=0$. 
Denote the set of the fixed points of the operator $F$ by

$$
\operatorname{Fix}(F)=\{u: F(u)=u\} .
$$

From (3.2), by substituting the new variables $\alpha^{3}=a,\left(v_{i}^{(n)}\right)^{3}=u_{i}^{(n)}$, for $i=1,4,5,8$. Then, one gets a new operator as follows:

$$
\left\{\begin{array}{c}
v_{1}^{(n+1)}=\frac{\alpha}{(b c)}\left((b c)^{2}\left(v_{1}^{(n)}\right)^{3}+\left(v_{4}^{(n)}\right)^{3}\right) \\
v_{4}^{(n+1)}=\frac{1}{\alpha(b c)}\left(b^{2}\left(v_{5}^{(n)}\right)^{3}+c^{2}\left(v_{8}^{(n)}\right)^{3}\right) \\
v_{5}^{(n+1)}=\frac{1}{\alpha(b c)}\left(\left(v_{1}^{(n)}\right)^{3}+(b c)^{2}\left(v_{4}^{(n)}\right)^{3}\right) \\
v_{8}^{(n+1)}=\frac{\alpha}{(b c)}\left(c^{2}\left(v_{5}^{(n)}\right)^{3}+b^{2}\left(v_{8}^{(n)}\right)^{3}\right) .
\end{array}\right.
$$

We deal with the fixed points of a system of recurrent equations given in (4.1). To this end, it suffices to solve the system of equations

$$
\left\{\begin{array}{l}
v_{1}=\frac{\alpha}{(b c)}\left((b c)^{2}\left(v_{1}\right)^{3}+\left(v_{4}\right)^{3}\right) \\
v_{4}=\frac{1}{\alpha(b c)}\left(b^{2}\left(v_{5}\right)^{3}+c^{2}\left(v_{8}\right)^{3}\right) \\
v_{5}=\frac{1}{\alpha(b c)}\left(\left(v_{1}\right)^{3}+(b c)^{2}\left(v_{4}\right)^{3}\right) \\
v_{8}=\frac{\alpha}{(b c)}\left(c^{2}\left(v_{5}\right)^{3}+b^{2}\left(v_{8}\right)^{3}\right)
\end{array}\right.
$$

Let us consider the following set:

$$
A=\left\{\left(v_{1}, v_{4}, v_{5}, v_{8}\right) \in \mathbb{R}_{+}^{4}: v_{1}=v_{8}, v_{4}=v_{5}, c=1\right\} .
$$

Note that the set $A$ is invariant with respect to $F$ i.e., $F(A) \subset A$.

Lemma 4.1. If a vector $\boldsymbol{u}$ is a fixed point of the operator $F$, then $\boldsymbol{u} \in M_{1}:=\left\{\boldsymbol{u}=\left(u_{1}, u_{4}, u_{5}, u_{8}\right) \in\right.$ $\left.\mathbf{R}_{+}^{4}: u_{1}=u_{8}, u_{4}=u_{5}\right\}$ or $\boldsymbol{u} \in M_{2}:=\left\{\boldsymbol{u}=\left(u_{1}, u_{4}, u_{5}, u_{8}\right) \in \mathbf{R}_{+}^{4}:\left(\sqrt[3]{u_{4}}+\sqrt[3]{u_{5}}\right)^{2}-\sqrt[3]{u_{4} u_{5}}=\right.$ $\left.\psi\left(\left(\sqrt[3]{u_{1}}+\sqrt[3]{u_{8}}\right)^{2}-\sqrt[3]{u_{1} u_{8}}\right)\right\}$, where $\psi(y)=\frac{b^{3}+\alpha y\left(1-b^{4}\right)}{b^{2} \alpha(b \alpha y-1)}$.

Proof. From the system (4.2), we have

$$
\begin{aligned}
& \left(v_{1}-v_{8}\right)\left(b \alpha\left(v_{1}^{2}+v_{1} v_{8}+v_{8}^{2}\right)-1\right)+\alpha b^{-1}\left(v_{4}-v_{5}\right)\left(v_{4}^{2}+v_{4} v_{5}+v_{5}^{2}\right)=0, \\
& (\alpha b)^{-1}\left(\left(v_{1}-v_{8}\right)\left(v_{1}^{2}+v_{1} v_{8}+v_{8}^{2}\right)+\left(v_{4}-v_{5}\right)\left(b^{2} v_{4}^{2}+b^{2} v_{4} v_{5}+b^{2} v_{5}^{2}+\alpha b\right)\right)=0 .
\end{aligned}
$$

From (4.4) and (4.5) one can conclude that if $v_{1}=v_{8}$ (respectively $v_{4}=v_{5}$ ), then, $v_{4}=v_{5}$ (respectively $\left.v_{1}=v_{8}\right)$. Therefore, $v_{4}=v_{5}$ if and only if $v_{1}=v_{8}$.

Now, let us assume that $v_{1} \neq v_{8}$ and $v_{4} \neq v_{5}$, then we can reduce the equations (4.4) and (4.5) to the following equation:

$$
\frac{\alpha b^{-1}\left(v_{4}^{2}+v_{4} v_{5}+v_{5}^{2}\right)}{\left(b \alpha\left(v_{1}^{2}+v_{1} v_{8}+v_{8}^{2}\right)-1\right)}=\frac{\left(b^{2} v_{4}^{2}+b^{2} v_{4} v_{5}+b^{2} v_{5}^{2}+\alpha b\right)}{\left(v_{1}^{2}+v_{1} v_{8}+v_{8}^{2}\right)} .
$$

Therefore, from the last equation we have

$$
v_{4}^{2}+v_{4} v_{5}+v_{5}^{2}=\frac{b^{3}+\alpha\left(v_{1}^{2}+v_{1} v_{8}+v_{8}^{2}\right)\left(1-b^{4}\right)}{b^{2} \alpha\left(b \alpha\left(v_{1}^{2}+v_{1} v_{8}+v_{8}^{2}\right)-1\right)}
$$

The equation (4.6) gives $\left(\sqrt[3]{u_{4}}+\sqrt[3]{u_{5}}\right)^{2}-\sqrt{3} u_{4} u_{5}=\psi\left[\left(\sqrt[3]{u_{1}}+\sqrt[3]{u_{8}}\right)^{2}-\sqrt[3]{u_{1} u_{8}}\right]($ see [9] $)$.

Remark 4.1. The fixed points of the operator $F$ belonging to the set $M_{2}$ give the ferromagnetic phases corresponding to the Ising model (2.2). It is analytically very difficult to examine the fixed points of the operator $F$ belonging to the set $M_{2}$. The ferromagnetic phase regions corresponding to the Ising model (2.2) can be numerically determined. 


\subsection{The existence of paramagnetic and ferromagnetic phases}

In this subsection, we analytically prove the existence of paramagnetic and ferromagnetic phases for the Ising model (2.2). Therefore, we deal with the fixed points of the operator $F$ belonging to the set $A$ given in the equation 4.3 .

Assume that $u_{1}^{(n)}=u_{8}^{(n)}, u_{4}^{(n)}=u_{5}^{(n)}$. After replacing $x_{n}=\frac{v_{1}^{(n)}}{v_{4}^{(n)}}$, we get the following system of recurrent equation

$$
x_{n+1}=\alpha^{2}\left(\frac{1+b^{2}\left(x_{n}\right)^{3}}{b^{2}+\left(x_{n}\right)^{3}}\right) \text {, }
$$

where $b>0, x_{n}>0$ and $\alpha>0$.

We will obtain the fixed points of the recurrent equation (4.7). To this end, it suffices to solve the following equation

$$
\alpha^{-2} x=f(x):=\left(\frac{1+b^{2} x^{3}}{b^{2}+x^{3}}\right)
$$

Note that in order to describe the phases (or the limiting Gibbs measures) corresponding to the model, we take into account the approach based on recurrent equations for partition functions (see [29, 31, 32, 42, 43] for details). We denote the set of the Gibbs measures corresponding to the Hamiltonian 2.2 by $\mathcal{G}_{H}$.

One can show that $f$ is bounded and thus the curve $y=\left(\frac{1+b^{2} x^{3}}{b^{2}+x^{3}}\right)$ must intersect the line $y=\alpha^{-2} x$. Therefore, our construction gives an element of $\mathcal{G}_{H}$. Note that if the equation (4.8) has more than one solution, then our construction gives more than one element of $\mathcal{G}_{H}$ (see [41, proposition 10.7] for details).

Proposition 4.2. The equation (4.8) has a unique solution if $0<b<1$. Assume that $b>\sqrt{2}$, then the equation (4.8) has 2 solutions if either $\eta_{1}(b)=\alpha^{-2}$ or $\eta_{2}(b)=\alpha^{-2}$. If $\eta_{1}(b)<\alpha^{-2}<\eta_{2}(b)$, then the equation (4.8) has 3 solutions. In fact, we have

$$
\eta_{i}(b)=\frac{1}{x_{i}}\left(\frac{1+b^{2} x_{i}^{3}}{b^{2}+x_{i}^{3}}\right),
$$

where $x_{i}$ are the solutions of the equation $b^{2} x^{6}-2\left(b^{4}-2\right) x^{3}+b^{2}=0$.

Proof. Let us take the first and the second derivatives of the function $f$, and we have

$$
\begin{gathered}
f^{\prime}(x)=\frac{3\left(b^{4}-1\right) x^{2}}{\left(b^{2}+x^{3}\right)^{2}}, \\
f^{\prime \prime}(x)=\frac{6\left(b^{4}-1\right) x\left(b^{2}-2 x^{3}\right)}{\left(b^{2}+x^{3}\right)^{3}} .
\end{gathered}
$$

From (4.9), assume that $b<1$ (with $x \geq 0$ ), then $f$ decreases and there exists a single solution of the equation $f(x)=\alpha^{-2} x$. Thus, in order to examine the phase transition of the model, we should solve the equation $f(x)=\alpha^{-2} x$ for $b>1$. One can show that the graph of $y=f(x)$ over interval $\left(0, \sqrt[3]{\frac{b^{2}}{2}}\right)$ is concave up, and the graph of $y=f(x)$ over the interval $\left(\sqrt[3]{\frac{b^{2}}{2}}, \infty\right)$ is concave down. As a result, there are at most 3 positive solutions for $f(x)=\alpha^{-2} x$.

According to Preston [41, proposition 10.7], the rational function $f$ has more than one fixed point if and only if equation $x f^{\prime}(x)=f(x)$ has more than one solution, which is the same as

$$
b^{2} x^{6}-2\left(b^{4}-2\right) x^{3}+b^{2}=0
$$




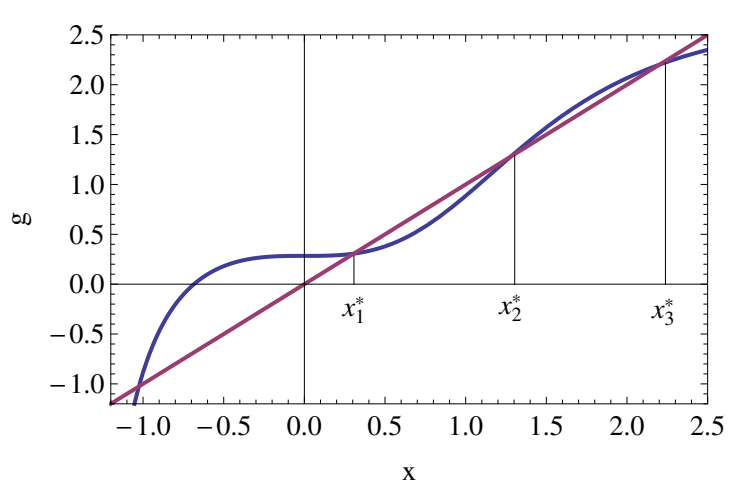

(a)

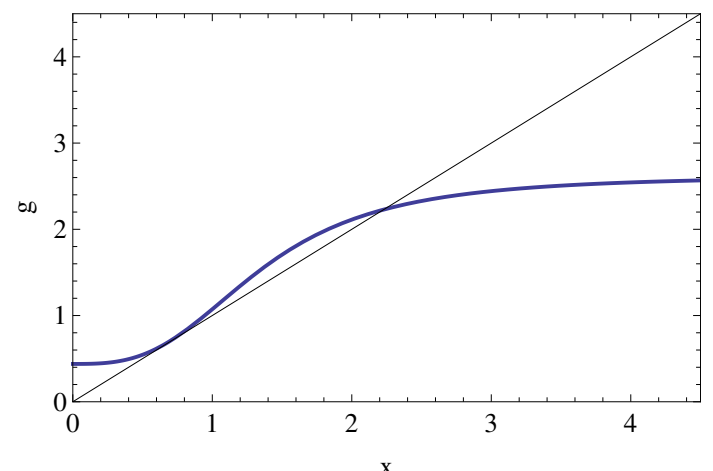

(b)

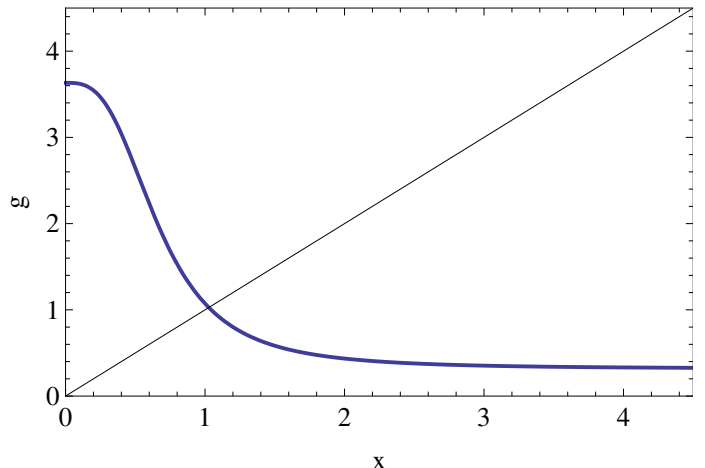

(c)

Figure 3. (Colour online) (a) There exist three positive roots of the equation 4.10 for $J=-4.5, J_{p}=$ $14, T=24.6$. (b) There exist two positive roots of the equation 4.10 for $J=2.6, T=24.6, J_{p}=11$. (c) There exists only one positive root of the equation 4.10 for $J=2.6, T=24.6, J_{p}=-15$.

These roots are

$$
x_{1}=\sqrt[3]{\frac{-2+b^{4}-\sqrt{4-5 b^{4}+b^{8}}}{b^{2}}}, x_{2}=\sqrt[3]{\frac{-2+b^{4}+\sqrt{4-5 b^{4}+b^{8}}}{b^{2}}} .
$$

Note that if $4-5 b^{4}+b^{8} \geqslant 0$, then the roots $x_{1}$ and $x_{2}$ are real numbers. In this case, $4-5 b^{4}+b^{8} \geqslant 0$ if and only if $b \in[0,1] \cap[\sqrt{2}, \infty)$.

\subsubsection{An illustrative example}

Let us consider the following equation:

$$
x=g(x):=\alpha^{2}\left(\frac{1+b^{2} x^{3}}{b^{2}+x^{3}}\right)
$$

From 4.10, one gets

$$
P_{4}(x)=x^{4}-\alpha^{2} b^{2} x^{3}+b^{2} x-\alpha^{2}=0 .
$$

To solve the equation (4.11) analytically is a rigorous and complicated problem. Therefore, we have manipulated the polynomial equation via Mathematica [44]. Here, we only deal with positive fixed points due to the positivity of exponential functions.

We have obtained at most 3 positive real roots for some parameters $J, J_{p}$ and $J_{t}=0$ (coupling constants) and temperature $T$. As seen in figure 3 (a), the equation (4.8) has 3 positive fixed roots for $J=-4.5, J_{p}=14, J_{t}=0, T=24.6\left(b=\mathrm{e}^{\frac{14}{24.6}}=1.77>\sqrt{2}\right)$. These all fixed points are $x_{0}^{*}=$ $-1.02554, x_{1}^{*}=0.306205, x_{2}^{*}=1.28008, x_{3}^{*}=2.20209$, respectively. 
One can examine that $g^{\prime}(0.306205)=0.219<1$ and $g^{\prime}(2.202)=0.59115<1$. Therefore, the fixed points $x_{1}^{*}=0.306205, x_{3}^{*}=2.20209$ are stable (attracting) fixed points. Moreover, due to $g^{\prime}(1.28008)=$ $1.3967>1$, the point $x_{2}^{*}=1.28008$ is an unstable (repelling) fixed point. The point $x_{c r}=1.15992$ is a breaking point of the function $g$. Thus, there are two extreme paramagnetic phases associated with the positive fixed points.

There exist two positive roots of the equation 4.10) for $J=2.6, T=24.6, J_{p}=11$ [figure 3 (b)]. There exists only one positive root for the equation 4.10 for $J=2.6, T=24.6, J_{p}=-15\left(b=\mathrm{e}^{\frac{-15}{24.6}}=0.54<1\right)$ [figure $3(\mathrm{c})]$.

\subsection{The periodic points of the operator $F$}

One of the most interesting problems in non-linear dynamic systems is to investigate the existence of periodic points. [45]. In statistical physics, these periodic points reveal the phase types corresponding to the given model [29, 32, 42].

We recall some definitions and results first.

Definition 4.3. A point $\boldsymbol{u}=\left(u_{1}, u_{4}, u_{5}, u_{8}\right)$ in $\mathbf{R}_{+}^{4}$ is called a periodic point of $F$ if there exists $p$ so that $F^{p}(\boldsymbol{u})=\boldsymbol{u}$ where $F^{p}$ is the pth iterate of $F$. The smallest positive integer $p$ satisfying the above is called the prime period or the least period of the point $\boldsymbol{u}$. Denote by $\operatorname{Per}_{p}(F)$ the set of periodic points with prime period $p$.

In order to find the periodic points of the operator $F$ with $p=2$ on $M_{1}$, we solve the equation $F(F(\mathbf{u}))=\mathbf{u}$. Therefore, we deal with the solutions of equation

$$
g(g(x))=x .
$$

Now, we consider the equation

$$
\frac{g(g(x))-x}{g(x)-x}=0
$$

After simple calculations, we get

$$
\begin{aligned}
& p_{6}(x):=4 b^{2}\left(1+b^{4} \alpha^{6}\right) x^{6}+\alpha^{2}\left(b^{4}-1\right) x^{5}+b^{2} \alpha^{4}\left(b^{4}-1\right) x^{4} \\
& +2 b^{4}\left(1+\alpha^{6}\right) x^{3}+b^{2} \alpha^{2}\left(b^{4}-1\right) x^{2}+\alpha^{4}\left(b^{4}-1\right) x+b^{2}\left(b^{4}+\alpha^{6}\right)=0 .
\end{aligned}
$$

In order to describe the periodic points with $p=2$ on $M_{1}$ of the operator $F$, we should find the solutions to (4.13) which are different from the solutions of the equation (4.11). In other words, we obtain the set

$$
M_{3}:=\left\{\left(u_{1}, u_{4}, u_{5}, u_{8}\right) \in \mathbf{R}_{+}^{4}: g(g(x))=x\right\} .
$$

Therefore, we should examine the roots of the polynomial $p_{6}(x)$ of degree 6 . As mentioned above, the roots of such polynomials can be described using the known formulae. In order to illustrate the problem, we have manipulated the equation (4.12) via Mathematica [44] [see figure 4(red color)]. The black graph in figure 4 represents the roots of the nonlinear function $y=g(x)$.

We can obtain an initial point of the sequence $\left(u_{1}^{(n)}, u_{4}^{(n)}, u_{5}^{(n)}, u_{8}^{(n)}\right)$ under positive boundary condition as follows:

$$
\mathbf{u}_{0}=\left(u_{1}^{(0)}, u_{4}^{(0)}, u_{5}^{(0)}, u_{8}^{(0)}\right)=\left(a b^{3}, \frac{b^{3}}{a}, \frac{1}{a b^{3}}, \frac{a}{b^{3}}\right) .
$$

In order to study some useful features of the function $g$, let us give the following lemma.

Lemma 4.4. 1) Let $b>1$, then the sequence $x_{n}=g\left(x_{n-1}\right)$ converges to $x_{0}=\sqrt[3]{\frac{u_{1}^{(0)}}{u_{4}^{(0)}}}=\alpha^{2}>0$ under a positive boundary condition, where $g$ is defined in (4.10).

2) Let $b<1$. then the sequence $y_{n}=h\left(y_{n-1}\right)$ converges to the initial point $y_{0}=\frac{\alpha^{2}\left(1+b^{2} \alpha^{6}\right)}{b^{2}+\alpha^{6}}>0$ under a positive boundary condition, where $h(x)=g(g(x))$.

The proof of lemma 4.4 is similar to [32]. 


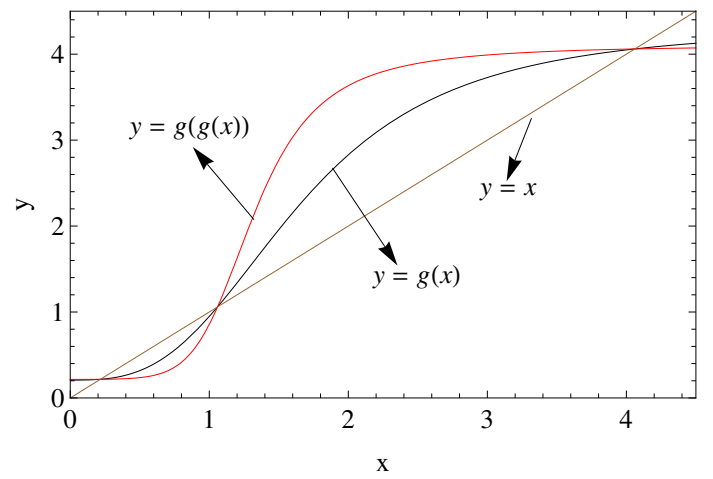

Figure 4. (Colour online) There exist three positive roots of the equation 4.8) (black color) for $J=-6$, $J_{p}=56.9, T=75$. Moreover, the equation 4.12 has three positive roots (red color) for $J=-6$, $J_{p}=56.9, T=75$.

\section{The phase diagrams of the model}

In this section, we plot the phase diagrams associated with our model in in three-parameter spaces. From the system 3.2 , the total partition function is given in terms of $\left(u_{i}\right)$ by

$$
Z^{(n)}=\left(u_{1}+u_{4}\right)^{3}+\left(u_{5}+u_{8}\right)^{3} .
$$

To discuss the phase diagram, we are interested in the following reduced variables:

$$
x^{(n)}=\frac{u_{4}^{(n)}+u_{5}^{(n)}}{u_{1}^{(n)}+u_{8}^{(n)}}, y^{(n)}=\frac{u_{1}^{(n)}-u_{8}^{(n)}}{u_{1}^{(n)}+u_{8}^{(n)}}, z^{(n)}=\frac{u_{4}^{(n)}-u_{5}^{(n)}}{u_{1}^{(n)}+u_{8}^{(n)}} .
$$

The variable $x^{(n)}$ is just a measure of the frustration of the nearest-neighbor bonds, and it is not an order parameter like $y^{(n)}, z^{(n)}$.

By using the equations 5.1 , we obtain the following equalities for our computation purpose in the next equations as

$$
\begin{gathered}
\frac{1+y^{(n)}}{2}=\frac{u_{1}^{(n)}}{u_{1}^{(n)}+u_{8}^{(n)}}, \frac{1-y^{(n)}}{2}=\frac{u_{8}^{(n)}}{u_{1}^{(n)}+u_{8}^{(n)}}, \\
\frac{x^{(n)}+z^{(n)}}{2}=\frac{u_{4}^{(n)}}{u_{1}^{(n)}+u_{8}^{(n)}}, \frac{x^{(n)}-z^{(n)}}{2}=\frac{u_{5}^{(n)}}{u_{1}^{(n)}+u_{8}^{(n)}} .
\end{gathered}
$$

From the equations (5.1) and from the last equations, after some lengthy and difficult calculations, we get the following recurrence dynamical system:

$$
\left\{\begin{array}{l}
x^{(n+1)}=\frac{\left(c^{2}\left(1-y^{(n)}\right)+b^{2}\left(x^{(n)}-z^{(n)}\right)\right)^{3}+\left(1+y^{(n)}+b^{2} c^{2}\left(x^{(n)}+z^{(n)}\right)\right)^{3}}{a^{2}\left(\left(x^{(n)}+b^{2} c^{2}\left(1+y^{(n)}\right)+z^{(n)}\right)^{3}+\left(b^{2}\left(1-y^{(n)}\right)+c^{2}\left(x^{(n)}-z^{(n)}\right)\right)^{3}\right)} \\
y^{(n+1)}=\frac{\left(x^{(n)}+b^{2} c^{2}\left(1+y^{(n)}\right)+z^{(n)}\right)^{3}-\left(b^{2}\left(1-y^{(n)}\right)+c^{2}\left(x^{(n)}-z^{(n)}\right)\right)^{3}}{\left(x^{(n)}+b^{2} c^{2}\left(1+y^{(n)}\right)+z^{(n)}\right)^{3}+\left(b^{2}\left(1-y^{(n)}\right)+c^{2}\left(x^{(n)}-z^{(n)}\right)\right)^{3}} \\
z^{(n+1)}=\frac{\left(c^{2}\left(1-y^{(n)}\right)+b^{2}\left(x^{(n)}-z^{(n)}\right)\right)^{3}-\left(1+y^{(n)}+b^{2} c^{2}\left(x^{(n)}+z^{(n)}\right)\right)^{3}}{a^{2}\left(\left(x^{(n)}+b^{2} c^{2}\left(1+y^{(n)}\right)+z^{(n)}\right)^{3}+\left(b^{2}\left(1-y^{(n)}\right)+c^{2}\left(x^{(n)}-z^{(n)}\right)\right)^{3}\right)},
\end{array}\right.
$$

we assume $a=\exp \left(\alpha^{-1}\right), b=\exp \left(-\alpha^{-1} \beta\right)$ and $c=\exp \left(-\alpha^{-1} \gamma\right)$, where $T / J=\alpha,-J_{p} / J=\beta,-J_{t} / J=\gamma$, respectively. 
The system obtained in $(5.2)$ is more complicated than one might have anticipated. Therefore, it remains difficult to be tackled analytically apart from simple limits and numerical methods that are necessary to study the behavior of the system in detail (see [2]).

Taking into account the positive boundary condition $\bar{\sigma}^{(n)}\left(V \backslash V_{n}\right) \equiv 1$, we obtain initial conditions

$$
\left\{\begin{array}{l}
x^{(1)}=\frac{1}{a^{2} c^{6}}, \\
y^{(1)}=\frac{b^{6}-1}{b^{6}+1}, \\
z^{(1)}=\frac{b^{6}-1}{a^{2} c^{6}\left(b^{6}+1\right)} .
\end{array}\right.
$$

For the fixed points, we consider the corresponding magnetization $m$ given by

$$
m^{(n)}=\frac{\left(1+x^{(n)}+y^{(n)}+z^{(n)}\right)^{3}-\left(1+x^{(n)}-y^{(n)}-z^{(n)}\right)^{3}}{\left(1+x^{(n)}+y^{(n)}+z^{(n)}\right)^{3}+\left(1+x^{(n)}-y^{(n)}-z^{(n)}\right)^{3}} .
$$

The initial point of the magnetization $m$ can be obtained as;

$$
m^{(1)}=\frac{\left(b^{2}-1\right)\left(\left(1+b^{2}\right)^{2}-b^{2}\right)\left(\left(1+b^{6}\right)^{2}-b^{6}\right)}{\left(1+b^{2}\right)\left(1-b^{2}+b^{4}\right)\left(1-b^{6}+b^{12}\right)} .
$$

In order to plot the phase diagrams, we iterate the recurrence equations (5.2) and (5.4) and observe the behavior of the phase diagrams after a large number of iterations $(n=10000)$.

A measure of the frustration of the nearest-neighbor bonds is determined by the variable $x$ [2]. Furthermore, if $u_{1}=u_{8}$ and $u_{4}=u_{5}$, that is $y^{(n)}=z^{(n)} \rightarrow 0$, then paramagnetic phase is obtained. If $y^{(n)} \nrightarrow 0$ or $z^{(n)} \nrightarrow 0$, then one obtains the ferromagnetic phase for the model (see [2, 5] for details).

In the simplest situation, a fixed point $\mathbf{u}^{*}=\left(u_{1}^{*}, u_{4}^{*}, u_{5}^{*}, u_{8}^{*}\right) \in \mathbf{R}_{+}^{4}$ is reached. Possible initial conditions with respect to different boundary conditions can be obtained in [2, 5]. Here, we consider initial conditions (5.3) and (5.5). Depending on $u_{1}^{*}, u_{4}^{*}, u_{5}^{*}, u_{8}^{*}$, in the simplest situation a fixed point $\left(x^{*}, y^{*}, z^{*}\right)$ is reached. It corresponds to a paramagnetic phase (briefly $\mathbf{P}$ ) if $y^{*}=0, z^{*}=0$ or to a ferromagnetic phase (briefly F) if $y^{*}, z^{*} \neq 0$. From formula of average magnetization (5.4) it follows that a situation where $y^{*}, z^{*} \neq 0$ but $m=0$, cannot occur. Otherwise, the system has periodic phases with the period $p$, i.e., the periodic phase is a configuration with some period.

Let us consider the limit

$$
\lim _{n \rightarrow \infty}\left(x^{(n)}, y^{(n)}, z^{(n)}\right)=\lim _{n \rightarrow \infty}\left(x^{(n+p)}, y^{(n+p)}, z^{(n+p)}\right)=\left(x^{(*)}, y^{(*)}, z^{(*)}\right) .
$$

The case $p=2$ corresponds to antiferromagnetic phase (briefly P2) and the case $p=4$ corresponds to the so-called antiphase (briefly P4), denoted by $<2>$ for compactness in [2, 5]. Finally, the system has aperiodic phases, that is $p>11$, the system has modulated phases (see [1, 4, 5] for details). We just consider periodic phases with period $p$ where $p \leqslant 12$ (briefly P2-P12). From the Lemma 4.1. we can obtain the following results:

- a paramagnetic phase: if $\mathbf{u}^{*} \in M_{1}$, in figure 5 (b), the white regions represent the paramagnetic phase. This represents the set $M_{1}$ given in Lemma 4.1;

- a ferromagnetic phase: if $\mathbf{u}^{*} \in M_{2}$, in figure 5](b), the red regions represent the ferromagnetic phase. This represents the set $M_{2}$ given in Lemma 4.1 ,

- in figure 5 (b), the yellow regions represent the $\mathbf{P 2}$ phase. This represents the set $M_{3}$ given in (4.14).

In figures 5 (a) and (b), we plotted the phase diagrams of the model on the third-order Cayley tree on the rectangular region $[-2,2] \times[-2,2]$.

In figure 5 (a), we observe that the phase diagram contains ferromagnetic (F), period-2, period-3 and modulated phases, though the paramagnetic phase (white region) completely disappears.

In the resultant phase diagram plotted by Vannimenus [2], the multicritical Libschit point is located in $\left(-J_{p} / J, T / J\right)=(1 / 3,0)$, that is, the phases defined as the ferromagnetic, paramagnetic, modulated and 

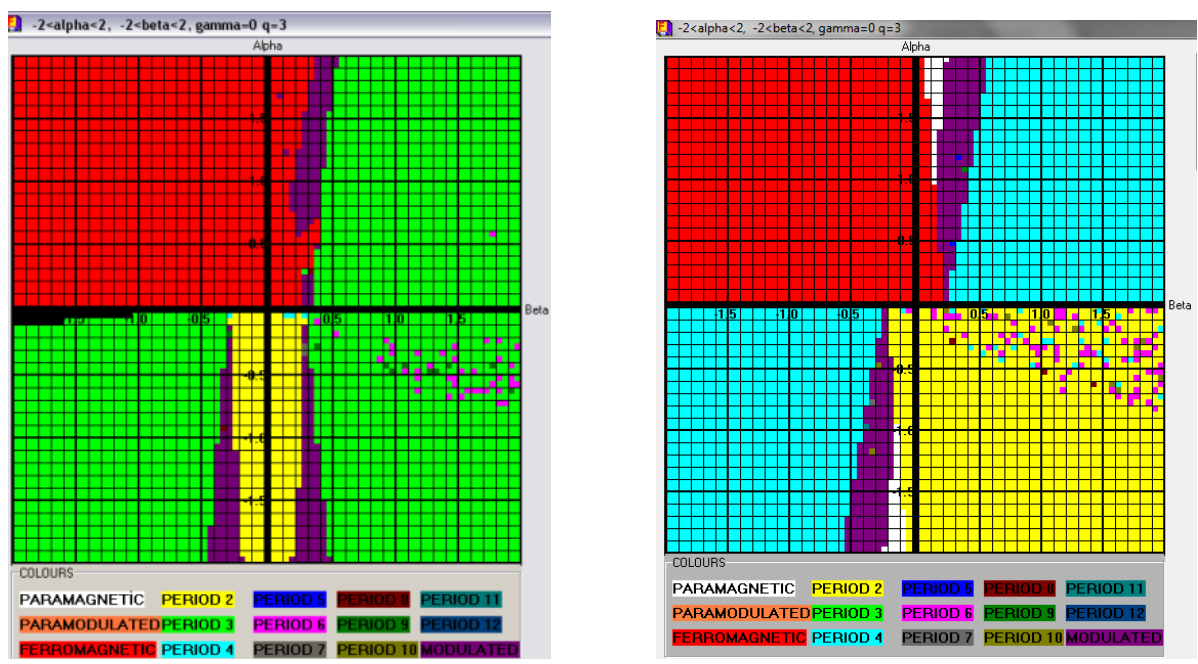

Figure 5. (Colour online) (a) Phase diagram of the model for $J_{p}=0$ (the left-hand figure); (b) Phase diagram of the model for $J_{t}=0$ (the right-hand figure).

$<2>$ meet at the point $\left(-J_{p} / J, T / J\right)=(1 / 3,0)$. In other words, all four phases meet at the multicritical point $(p=1 / 3, T=0)$. Contrary to Vannimenus's work [2], here the multicritical Libschit points appear in non-zero points. In the resultant phase diagram in figure 5 (b), the ferromagnetic, paramagnetic, modulated and $<2>$ phases intersect at the point $\left(-J_{p} / J, T / J\right)=(0.15,0.39)$. Moreover, the phases of ferromagnetic, paramagnetic, modulated and $\mathbf{P} 2$ intersect at the multicritical point $\left(J_{p} / J,-T / J\right)=$ $(-0.15,-0.39)$.

In figure 5 (b), the phase diagram consists of ferromagnetic $(\mathrm{F})$, paramagnetic $(\mathrm{P})$ (fixed point), chaotic (C) (or modulated), and antiferromagnetic + + - - (four cycle antiferromagnetic phase) phases.

In the regions we have called the modulated $(\mathbf{M})$ phase, narrow regions with periodic orbit representing commensurate phases have been identified. It is really difficult to detect those with a periodicity higher than 12 in the modulated phase regions. In order to determine the types of these phases, Lyapunov exponent and the attractors are analyzed for some critical parameters (see [1, 4, 5, 16]). The modulated phases may generally contain two phases that correspond to the devil's staircase [5]. These are called commensurate (periodic) and incommensurate (aperiodic) regions, respectively.

In Proposition 4.2, we analytically showed that there exits a paramagnetic phase if and only if $b \in[0,1] \cap[\sqrt{2}, \infty)$. Therefore, we can present the following theorem.

Theorem 5.1. The model 2.2) (with $x \geqslant 0, \alpha>0, b>0$ ) has a unique paramagnetic phase (Gibbs measure) if $b<1$. Assume that if $b>\sqrt{2}$, then the model (2.2) has exactly two paramagnetic phases if either $\eta_{1}(b)=\alpha^{-2}$ or $\eta_{2}(b)=\alpha^{-2}$. If $\eta_{1}(b)<\alpha^{-2}<\eta_{2}(b)$, then the model 2.2) has exactly three paramagnetic phases.

The proof of theorem 5.1 is obtained from the proposition (4.2). Note that from the equation (5.1), the corresponding phase is paramagnetic, because we take into account $u_{1}^{(n)}=u_{8}^{(n)}$ and $u_{4}^{(n)}=u_{5}^{(n)}$, that is, $x^{(n)} \rightarrow x^{*}, y^{(n)} \rightarrow 0$ and $z^{(n)} \rightarrow 0$ (see [2] for details).

Remark 5.1. We conclude that the phase boundaries between the periodic phases presented in the diagrams are only approximate, because they are based on the iteration of recursion relations in equation (5.2), rather than on the minimization of the free energy. Therefore, it is possible to calculate the free energy via the scheme proposed by Gujrati [46]. Therefore, one can consider employing that scheme in order to improve the precision of the phase diagrams. To calculate the free energy will not be considered here.

Remark 5.2. In order to distinguish these phases from each other, one needs to analyse the modulated phase regions via Lyapunov exponent and the attractors in detail (see [1, 4, 5, 16]). Here, we do not give these details. 


\subsection{The fixed points of the operator $F$ for $b=1$}

In the equation (4.2), if we assume as $b=1$, then we have

$$
\left\{\begin{array}{l}
v_{1}=\frac{\alpha}{c}\left(c^{2} v_{1}^{3}+v_{4}^{3}\right) \\
v_{4}=\frac{1}{\alpha c}\left(v_{5}^{3}+c^{2} v_{8}^{3}\right) \\
v_{5}=\frac{1}{\alpha c}\left(v_{1}^{3}+c^{2} v_{4}^{3}\right) \\
v_{8}=\frac{\alpha}{c}\left(c^{2} v_{5}^{3}+v_{8}^{3}\right)
\end{array}\right.
$$

Now, we describe the positive fixed points of system 5.6. Let us consider the following set

$$
B:=\left\{\left(v_{1}, v_{4}, v_{5}, v_{8}\right) \in \mathbf{R}_{+}^{4}: v_{1}=v_{8}, v_{4}=v_{5}\right\} .
$$

Remark 5.3. From the system (5.6), it is clear that the equations $v_{1}=v_{8}$ and $v_{4}=v_{5}$ do not satisfy, i.e., the set $B$ given in 5.7 is empty. Therefore, the paramagnetic phase regions in the phase diagrams associated with the model disappear in $[-2,2] \times[-2,2] \subset \mathbf{R}^{2}$.

We can conclude that there exists at least one fixed point of the corresponding $g$ function if $u_{1}=$ $u_{8}, u_{4}=u_{5}$ and $c=1$, that is, the system has a parametric phase [see figure 5](b)]. Conversely, if $b=1$ then $u_{1}=u_{8}, u_{4}=u_{5}$ are not satisfied, so the corresponding operator has no fixed points. In this case, the system has no paramagnetic phase. However, the same system has a ferromagnetic phase [see figure 5 (a)].

\section{Conclusions}

Written for both mathematics and physics audience, this paper has a fourfold purpose:

1) to analytically study the recurrence equations associated with the model 2.2);

2) to numerically obtain the paramagnetic, the ferromagnetic and period 2 regions corresponding to the sets $M_{1}, M_{2}, B$, respectively;

3) to illustrate the fixed points of the corresponding operator;

4) to compare the numerical results with the exact solutions of the model.

We state some unsolved problems that turned out to be rather complicated and require a further consideration:

1. Do any other invariant sets of the operator $F$ exist?

2. Do positive fixed points of the operator $F$ exist outside the invariant sets?

3. Does there exist a periodic point $(p>2)$ of rather cumbersome high-order equations that can be solved using analytic methods?

In the first case, we have already obtained the fixed points of the operator $F$ so that $u_{1}=u_{8}$ and $u_{4}=u_{5}$. For the periodic case, however, it is not possible to obtain all solutions satisfying all requirements of equations (3.2), so that $\left\{\mathbf{u}=\left(u_{1}, u_{4}, u_{5}, u_{8}\right) \in \mathbf{R}_{+}^{4}: F^{p}(\mathbf{u})=\mathbf{u}, p>1\right\}$ is invariant. The proof of this statement involves a number mathematical complexities. Moreover, in the second case $(b=1)$, it is much more difficult to analytically find the fixed points of the operator $F$.

Vannimenus [2] showed that at $T=0$, only two different ground-states are encountered: the ferromagnetic state, for $p\left(=-J_{p} / J\right)$ smaller than $1 / 3$, and a state of period 4 in which the magnetization of the successive generations alternates with an antiferromagnetic structure $(++--)$, for $p>1 / 3$. By using the standard approach, we have analytically proved that there exits a paramagnetic phase when $J_{p}>0$ and for the phase with period 2 when $J_{p}<0$. Our results do not contrast with those of Vannimenus [2]. In this present paper, we show that a paramagnetic phase completely disappears at $T=0$. We plan to experiment in our future work in order to analyse the same problem analytically for the high-order Cayley 
tree. Recently, the author [47] has studied the existence of the Gibbs measures of an Ising model with competing interactions on the triangular chandelier-lattice. The phase diagrams corresponding to the Gibbs states on the Cayley-like lattices have not been examined yet. In the future papers, we are planning to investigate the same problems (see [40]).

\section{Acknowledgements}

The author thanks the referee for the careful reading of the manuscript and for the valuable comments and suggestions.

\section{References}

1. Inawashiro S., Thompson C.J., Honda G., J. Stat. Phys., 1983, 33, 419-436, doi 10.1007/BF01009804

2. Vannimenus J., Z. Phys. B: Condens. Matter, 1981, 43, No. 2, 141-148, doi 10.1007/BF01293605.

3. Ganikhodjaev N.N., Akın H., Uguz S., Temir T., J. Stat. Mech: Theory Exp., 2011, 03, P03025, doi $10.1088 / 1742-5468 / 2011 / 03 / \mathrm{P} 03025$

4. Uguz S., Ganikhodjaev N.N., Akın H., Temir S., Int. J. Mod. Phys. C, 2012, 23, No. 5, 1250039, doi $10.1142 / \mathrm{S} 0129183112500398$

5. Mariz M., Tsalis C., Albuquerque A.L., J. Stat. Phys., 1985, 40, 577-592, doi 10.1007/BF01017186.

6. Yokoi C.S.O., de Oliveira M.J., Salinas S.R., Phys. Rev. Lett., 1985, 54, No. 3, 163-166, doi $10.1103 /$ PhysRevLett.54.163.

7. Lebowitz J.L., J. Stat. Phys., 1977, 16, No. 6, 463-476, doi 10.1007/BF01152284

8. Ganikhodjaev N.N., Akın H., Uguz S., Temir S., J. Concr. Appl. Math., 2011, 9, No. 1, 26-34.

9. Ganikhodjaev N.N., Uguz S., Physica A, 2011, 390, No. 23-24, 4160-4173, doi 10.1016/j.physa.2011.06.044

10. Ganikhodjaev N.N., Temir S., Akın H., J. Stat. Phys., 2009, 137, 701-715, doi 10.1007/s10955-009-9869-z.

11. Ganikhodjaev N.N., Akın H., Uguz S., Temir S., Phase Transitions, 2011, 84, No. 11-12, 1045-1063, doi $10.1080 / 01411594.2011 .579395$

12. Akın H., Uguz S., Temir S., AIP Conf. Proc., 2010, 1281, 607-611, doi $10.1063 / 1.3498550$

13. Uguz S., Akın H., Chin. J. Phys., 2011, 49, No. 3, 788-801.

14. Uguz S., Akın H., Physica A, 2010, 389, 1839, doi:10.1016/j.physa.2009.12.057

15. Uguz S., Ganikhodjaev N.N., Akın H., Temir S., Acta Phys. Pol. A, 2012, 121, No. 1, 114-118, doi $10.12693 /$ APhysPolA.121.114

16. Inawashiro S., Thompson C.J., Phys. Lett. A, 1983, 97, 245-248, doi 10.1016/0375-9601(83)90758-2

17. Moraal H., Physica A, 1978, 92, 305-314, doi 10.1016/0378-4371(78)90037-7

18. Chakraborty K.G., J. Magn. Magn. Mater., 1992, 114, No. 1-2, 155-160, doi:10.1016/0304-8853(92)90340-T

19. Horiguchi T., Physica A, 1986, 136, No. 1, 109-123, doi 10.1016/0378-4371(86)90045-2

20. Azhari M., Benayad N., Mouhib M., Phase Transitions, 2017, 90, No. 5, 485-499, doi 10.1080/01411594.2016.1227985

21. Akın H., Saygılı H., AIP Conf. Proc., 2015, 1676, 020026, doi 10.1063/1.4930452.

22. Akın H., Rozikov U.A., Temir S., J. Stat. Phys., 2011, 142, No. 2, 314-321, doi:10.1007/s10955-010-0106-6.

23. Nazarov Kh.A., Rozikov U., Theor. Math. Phys., 2003, 135, No. 3, 881-888, doi:10.1023/A:1024091206594

24. Akın H., Ganikhodjaev N., Uguz S., Temir S., AIP Conf. Proc., 2011, 1389, No. 1, 2004-2007, doi $10.1063 / 1.3637008$

25. Akın H., Ganikhodjaev N.N., Temir S., Uguz S., Acta Phys. Pol. A, 2013, 123, No. 2, 484-487, doi 10.12693/APhysPolA.123.484

26. Akin H., Int. J. Mod. Phys. B, 2017, 31, No. 13, 1750093, doi 10.1142/S021797921750093X

27. Akın H., Chin. J. Phys., 2016, 54, No. 4, 635-649, doi 10.1016/j.cjph.2016.07.010.

28. Akın H., Int. J. Mod. Phys. C, 2018, 29, No. 2, 1850016, doi:10.1142/S012918311850016X

29. Akın H., Temir S., Condens. Matter Phys, 2011, 14, No. 2, 23003, doi $10.5488 / C M P .14 .23003$

30. Bleher P.M., Ruiz J., Zagrebnov V.A., J. Stat. Phys., 1995, 79, 473-482, doi $10.1007 /$ BF02179399

31. Bleher P.M., Ganikhodjaev N.N., Theory Probab. Appl., 1990, 35, 216-227, doi $10.1137 / 1135031$

32. Rozikov U.A., Akın H., Uguz S., Math. Phys. Anal. Geom., 2014, 17, 103-114, doi 10.1007/s11040-014-9144-7

33. Rozikov U.A., Gibbs Measures on Cayley Trees, World Scientific, Singapore, 2013.

34. Bleher P., Zalys E., Lith. Math. J., 1988, 28, No. 2, 127.

35. Bleher P.M., Commun. Math. Phys., 1990, 128, No. 2, 411-419, doi $10.1007 /$ BF02108787

36. Ganikhodjaev N.N., Akın H., Temir T., Turk. J. Math., 2007, 31, No. 3, 229-238. 
37. Gandolfo D., Ruiz J., Shlosman S., J. Stat. Phys., 2012, 148, 999-1005, doi 10.1007/s10955-012-0574-y

38. Kindermann R., Snell J.L., Markov Random Fields and Their Applications, Amer. Math. Soc., Providence, Rhode Island, 1980.

39. Mukhamedov F., Akın H., Khakimov O., J. Stat. Mech., 2017, 2017, 053208, doi 10.1088/1742-5468/aa6c88.

40. Akın H., (unpublished), URL https://www.researchgate.net/publication/340540145

41. Preston Ch.J., Gibbs States on Countable Sets, Cambridge Univ. Press, Cambridge, 1974.

42. Ganikhodjaev N., Temir S., Akin H., CUBO, A Mathematical J., 2005, 7, No. 3, 39-48.

43. Ganikhodjaev N., Rahmatullaev A., Phase Transitions, 2019, 92, No. 8, 730-736, doi $10.1080 / 01411594.2019 .1639700$

44. Wolfram Research, Inc., Mathematica, Version 8.0, Champaign, IL, 2010.

45. Ganikhodjaev N.N., Rozikov U.A., Theor. Math. Phys., 1997, 111, 480-486, doi 10.1007/BF02634202.

46. Gujrati P.D., Phys. Rev. Lett., 1995, 74, 809, doi:10.1103/PhysRevLett.74.809

47. Akın H., Condens. Matter Phys., 2019, 22, No. 2, 23002, 1-14, doi 10.5488/CMP.22.23002

\title{
Визначення парамагнітної та феромагнітної фаз моделі Ізінга на дереві Кейлі третього порядку
}

\author{
Г. Акін \\ вул. Джейхун Атуф Кансу 1164, Сокак, 9/4, TR06105, Чанкая, Анкара, Туреччина \\ У статті отримано рекурентні рівняння моделі Ізінга з трьома константами зв'язку на дереві Кейлі тре- \\ тього порядку. Представлено характеристики парамагнітна та феромагнітної фази, пов'язані з моделлю \\ Ізінга. Строго вивчаються типи фаз та статистична сума, що відповідають моделі. Точні розв'язки згада- \\ ної моделі порівнюються з числовими результатами, наведеними в Ganikhodjaev et al. [ J. Concrete and \\ Applicable Mathematics, 2011, 9, No. 1, 26-34].
}

Ключові слова: дерево Кейлі, модель Ізінга, парамагнітна фаза, феромагнітна фаза 


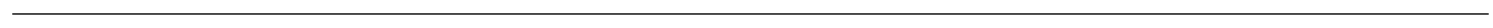

